\title{
Methylation of the KEAP1 gene promoter region in human colorectal cancer
}

\author{
Naoyuki Hanada 1,2, Takenori Takahata', Qiliang Zhou' ${ }^{1}$ Xulu Ye ${ }^{1}$, Ruowen Sun ${ }^{1,5}$, Jugoh Itoh ${ }^{1}$, Atsushi Ishiguro ${ }^{1}$, \\ Hiroshi Kijima ${ }^{3}$, Junsei Mimura ${ }^{4}$, Ken Itoh ${ }^{4}$, Shinsaku Fukuda ${ }^{2}$ and Yasuo Saijo ${ }^{1^{*}}$
}

\begin{abstract}
Background: The Keap1-Nrf2 pathway has been reported to be impaired in several cancers. However, the status of Keap1-Nrf2 system in human colorectal cancer (CRC) has not been elucidated.

Methods: We used colorectal cancer (CRC) cell lines and surgical specimens to investigate the methylation status of the KEAP1 promoter region as well as expression of Nrf2 and its downstream antioxidative stress genes, NQO-1 and $A K R 1 C 1$.

Results: DNA sequencing analysis indicated that all mutations detected were synonymous, with no amino acid substitutions. We showed by bisulfite genomic sequencing and methylation-specific PCR that eight of 10 CRC cell lines had hypermethylated $\mathrm{CPG}$ islands in the KEAP1 promoter region. HT29 cells with a hypermethylated KEAP1 promoter resulted in decreased mRNA and protein expression but unmethylated Colo320DM cells showed higher expression levels. In addition, treatment with the DNA methyltransferase inhibitor 5-Aza-dC combined with the histone deacetylase inhibitor trichostatin A (TSA) increased KEAP1 mRNA expression. These result suggested that methylation of the KEAP1 promoter regulates its mRNA level. Time course analysis with the Nrf2-antioxidant response element (ARE) pathway activator t-BHQ treatment showed a rapid response within $24 \mathrm{~h}$. HT29 cells had higher basal expression levels of NQO-1 and AKR1C1 mRNA than Colo320DM cells. Aberrant promoter methylation of KEAP1 was detected in 53\% of tumor tissues and 25\% of normal mucosae from 40 surgical CRC specimens, indicating that cancerous tissue showed increased methylation of the KEAP1 promoter region, conferring a protective effect against cytotoxic anticancer drugs.
\end{abstract}

Conclusion: Hypermethylation of the KEAP1 promoter region suppressed its mRNA expression and increased nuclear Nrf2 and downstream ARE gene expression in CRC cells and tissues.

\section{Background}

Colorectal cancer $(\mathrm{CRC})$ is the second leading cause of cancer-related deaths in most Western countries [1]. Over the past decade, molecular-targeted drugs have been applied in combination with cytotoxic agents. Consequently, the median overall survival for patients with advanced CRC has become longer than 24 months. Although the spectrum of therapeutic agents is becoming broader, many issues remain to be solved regarding cancer progression and acquisition of resistance to chemotherapy in CRC.

\footnotetext{
* Correspondence: yasosj@cc.hirosaki-u.ac.jp

'Department of Medical Oncology, Hirosaki University Graduate School of Medicine, 5 Zaifu-cho, Hirosaki 036-8562, Japan

Full list of author information is available at the end of the article
}

The Kelch-like ECH-associated protein 1 (Keap1) and nuclear factor-erythroid 2 -related factor 2 (Nrf2) pathway is one of the master regulators of cellular defense against oxidative and electrophilic stresses [2-4]. Nrf2 is a basic region-leucine zipper (bZip)-type transcription factor, which was identified as a binding protein of the $\beta$-globin gene locus [5,6]. Subsequently, Nrf2 was recognized to be a major transactivation factor for antioxidant response element (ARE)-dependent gene transcription [7]. The ARE is a cis-acting regulatory element of genes encoding phase II detoxification enzymes and antioxidant proteins, such as $\mathrm{NAD}(\mathrm{P}) \mathrm{H}$ quinone oxidoreductase-1 (NQO-1), glutathione S-transferases (GST), heme oxygenase-1 (HO-1), and aldo-keto reductase family 1 member C1 (AKR1C1). Keap1 is a negative regulator of Nrf2 and its main function is to serve as an adaptor for

\section{Biomed Central}


cullin3/ring box1 (Cul3/Rbx1) E3 ubiquitin ligase complex [8-12]. Under physiological conditions, Keap1 maintains a low basal level of Nrf2 by constantly targeting Nrf2 for ubiquitin-mediated protein degradation $[13,14]$. Once a cell is exposed to oxidative stress, Keap1 acts as a sensor and its cysteine residues are modified. This modification prevents rapid degradation of Nrf2, and the accumulated Nrf2 translocates into the nucleus, leading to active transcription of downstream cytoprotective genes.

The Keap1-Nrf2 signaling pathway is impaired in lung cancer, which is caused by mutations within functionally important domains of the KEAP1 or NRF2 gene [15-17]. Impaired Keap1 activity and somatic mutation of Nrf2 lead to full Nrf2 activation, and cancer cells may acquire a protective mechanism against the surrounding microenvironment, resulting in cancer cell proliferation, differentiation, and chemoresistance $[15,17]$. Similar KEAP1 mutations have been reported in patients with gall bladder cancer and in breast cancer cell lines $[18,19]$.

Recently, Wang et al. reported that the promoter region of KEAP1 is aberrantly hypermethylated and KEAP1 mRNA expression levels are low in some lung cancer cell lines and lung cancer tissues [20]. Aberrant methylation of the KEAP1 promoter region was also reported in prostate cancer [21] and malignant glioma [22]. However, the methylation status of KEAP1 in CRC has not been elucidated.

As an impaired Keap1-Nrf2 system is induced by mutation or hypermethylation in several types of human cancer, we hypothesized that mutation or epigenetic changes of KEAP1 may decrease Keap1 expression and increase Nrf2 activity and transactivation of its downstream genes in CRC. In the present study, we investigated the methylation status of KEAP1 in 10 CRC cell lines and 40 surgically excised CRC tissue specimens. We found frequent hypermethylation of the KEAP1 gene promoter region in human $\mathrm{CRC}$. In addition, the levels of Nrf2 target gene expression were upregulated in hypermethylated cells.

\section{Methods}

\section{CRC cell lines and patient tissue samples}

Human CRC cell lines were obtained from cell banks. The HT29 cell line was from American Type Culture Collection, while WiDr, LoVo, DLD-1, SW837, and Colo320DM cell lines were from the Human Science Research Resources Bank (Osaka, Japan). HCT15 and SW480 were from the Cell Resource Center for Biomedical Research Institute of Development, Aging, and Cancer, Tohoku University. TT1TKB and CW-2 were from RIKEN BioResource Center (Ibaraki, Japan). HT29, WiDr, LoVo, DLD-1, SW480, and SW837 were cultured in Dulbecco's Modified Eagle's Medium (DMEM) containing 10\% heat-inactivated fetal bovine serum (FBS). HCT15, CW-2, and Colo320DM were cultured in RPMI1640 medium containing 10\% FBS. Forty CRC tissues and adjacent normal colorectal tissue samples were collected with written informed consent at Hirosaki University Hospital. The tissues were immediately frozen and stored at $-80^{\circ} \mathrm{C}$ after surgical resection. The study of CRC tissues samples was approved by the Ethics Committee of Hirosaki University School of Medicine.

\section{Cell treatment}

HT29 cells were plated at $5 \times 10^{6}$ cells $/ 10$-cm dish $24 \mathrm{~h}$ prior to treatment. Cells were treated with $10 \mu \mathrm{M} 5$-aza2'-deoxycytidine (5-Aza-dC) for 96 h to block CpG methylation, followed by treatment with $1 \mu \mathrm{M}$ trichostatin A (TSA), a reversible inhibitor of histone deacetylase, for $24 \mathrm{~h}$. To evaluate downstream gene expression of Nrf2, HT29 and Colo320DM cells were treated with 50 $\mu \mathrm{M}$ tert-butylhydroquinone ( $\mathrm{t}-\mathrm{BHQ})$, a potent inducer of Nrf2-dependent gene expression, and cells were harvested at $2,4,8,12$, and $24 \mathrm{~h}$ after treatment. RNA was then extracted, and real-time reverse transcription-polymerase chain reaction (RT-PCR) was performed as described below to measure the expression of NQO1 and $A K R 1 C 1$. The TaqMan Gene Expression Assay ID for the NQO1 mRNA is Hs00168547_m1, and that for AKR1C1 is Hs00413886_m1.

\section{DNA and RNA extraction and DNA sequencing of the KEAP1 gene}

Genomic DNA was extracted from CRC cell lines using a QIAmp DNA Mini kit (Qiagen, Valencia, CA), and RNA was isolated using an RNeasy kit (Qiagen) according to the manufacturer's protocols. The DNA/RNA concentration and their quality were evaluated by measuring the ratio of optical density at $260 / 280 \mathrm{~nm}$ with NanoDrop (NanoDrop Technologies Wilmington, DE). For detection of KEAP1 mutation, DNA extracted from cell lines was amplified using AmpliTaq Gold ${ }^{\circledR}$ Fast PCR Master Mix (Applied Biosystems, Carlsbad, CA). Direct sequencing was performed using the primer sets reported previously by Shibata et al. [18].

\section{Methylation-specific PCR (MSP) and bisulfite sequencing PCR (BSP) of the KEAP1 gene}

The primer sets of MSP and BSP used to target the $\mathrm{CpG}$ islands located in the putative promoter region of KEAP1 [20] are shown in Table 1 and Figure 1. These primer sets were designed using Methyl Primer Express Software v1.0 (Applied Biosystems), and PCR conditions for MSP and BSP are shown in Table 1. Aliquots of 2 $\mu \mathrm{g}$ of extracted DNA from CRC cell lines were 
Table 1 PCR primers and thermal cycling conditions

\begin{tabular}{|c|c|c|}
\hline Methods & Primers & Sequence \\
\hline \multirow[t]{2}{*}{ BSP } & & Forward: 5'-AAGAAAAGAAAAGAAAAGAAAATTTAG-3' \\
\hline & & Reverse: 5'-TTAAGTGAGGTAGATAATTTाTT-3' \\
\hline $\begin{array}{l}\text { PCR } \\
\text { conditions }\end{array}$ & & $\begin{array}{l}\text { Initial denaturation at } 95^{\circ} \mathrm{C}(10 \mathrm{~min}) \text { and } 35 \text { cycles at } 95^{\circ} \mathrm{C}(3 \mathrm{~s}), 52^{\circ} \mathrm{C}(3 \mathrm{~s}), 72^{\circ} \mathrm{C}(5 \mathrm{~s}) \text {, and a final extension at } 72^{\circ} \mathrm{C}(10 \\
\text { s) }\end{array}$ \\
\hline \multirow[t]{2}{*}{ MSP } & Methylation- & Forward: 5'-TAGATAATTITTITAAGATTTTGCGGTCG-3' \\
\hline & Specific & Reverse: 5'-TCCTCGCGAAACTACGC-3' \\
\hline $\begin{array}{l}\text { PCR } \\
\text { condition }\end{array}$ & & $\begin{array}{l}\text { Initial denaturation at } 95^{\circ} \mathrm{C}(10 \mathrm{~min}) \text { and annealing temperature decrement of } 0.5^{\circ} \mathrm{C} \text { every cycle (from } 70^{\circ} \mathrm{C} \text { to } 66.5^{\circ} \mathrm{C} \text { ) } \\
\text { followed by } 32 \text { cycles of } 66^{\circ} \mathrm{C}(3 \mathrm{~s}), 72^{\circ} \mathrm{C}(5 \mathrm{~s}) \text {, and a final extension at } 72^{\circ} \mathrm{C}(10 \mathrm{~s})\end{array}$ \\
\hline \multirow[t]{2}{*}{$\overline{M S P}$} & $\begin{array}{l}\text { Non- } \\
\text { methylation }\end{array}$ & Forward: 5'-TAGATAATTTTTITTAGATTTTGTGGTTG-3' \\
\hline & -specific & Reverse: 5'-TCCTCACAAAACTACAC-3' \\
\hline $\begin{array}{l}\text { PCR } \\
\text { condition }\end{array}$ & & $\begin{array}{l}\text { Initial denaturation at } 95^{\circ} \mathrm{C}(10 \mathrm{~min}) \text { and annealing temperature decrement of } 0.5^{\circ} \mathrm{C} \text { every cycle (from } 64^{\circ} \mathrm{C} \text { to } 60.5^{\circ} \mathrm{C} \text { ) } \\
\text { followed by } 32 \mathrm{cycles} \text { of } 60^{\circ} \mathrm{C}(3 \mathrm{~s}), 72^{\circ} \mathrm{C}(5 \mathrm{~s}) \text {, and a final extension at } 72^{\circ} \mathrm{C}(10 \mathrm{~s})\end{array}$ \\
\hline
\end{tabular}

converted using an Epitect Bisulfite kit (Qiagen) in accordance with the manufacturer's instructions. Direct DNA sequencing by dye terminator cycle sequencing was performed after bisulfite treatment using an ABI 310 Genetic analyzer (Applied Biosystems). PCR amplification with MSP primers was then performed using 10 $\mu \mathrm{l}$ of AmpliTaq Gold ${ }^{\circledR}$ Fast PCR Master Mix and $20 \mathrm{ng}$ of template DNA (the PCR conditions are shown in Table 1). CpG-methylated HeLa genomic DNA and 5Aza-dC-treated Jurkat genomic DNA (New England Biolabs Japan, Tokyo, Japan) were used as controls for methylated and unmethylated sequence detection, respectively. MSP products were analyzed by $2 \%$ agarose gel electrophoresis, stained with ethidium bromide, and visualized with a UV transilluminator.

\section{Real-time RT-PCR}

Expression of KEAP1 mRNA was measured by quantitative real-time PCR in triplicate using TaqMan Gene Expression Assays (Applied Biosystems) in the ABI PRISM 7000 sequence detection system (Applied Biosystems). Intact total RNA was extracted as described above. Reverse transcriptase reactions were performed on aliquots of $2 \mu \mathrm{g}$ of total RNA using a High Capacity cDNA Reverse Transcription kit (Applied Biosystems) according to the manufacturer's protocol. The

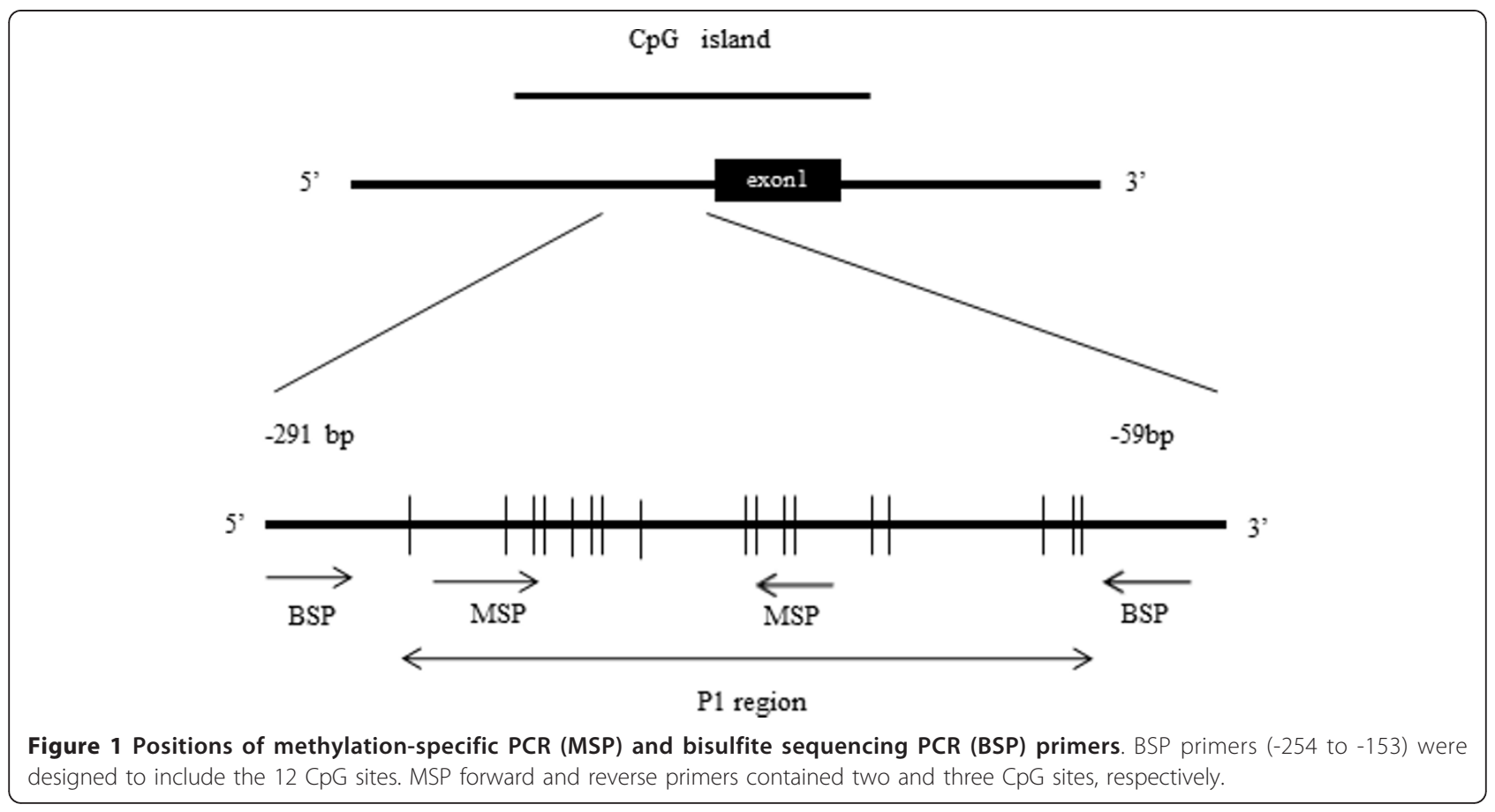


conditions for reverse transcription were $25^{\circ} \mathrm{C}(10 \mathrm{~min})$, $37^{\circ} \mathrm{C}(120 \mathrm{~min})$, and $85^{\circ} \mathrm{C}(5 \mathrm{~min})$. The TaqMan Gene Expression Assay ID of the KEAP1 mRNA is Hs00202227_m1. Calculations were performed using the comparative $\mathrm{C}_{\mathrm{T}}$ method. GAPDH (Assay ID Hs99999905_m1) was used as an endogenous control gene for normalization of PCR for the amount of RNA added to the reverse transcription reactions. The mRNA levels are expressed as fold induction relative to the control. The conditions for real-time PCR were $50^{\circ} \mathrm{C}(2$ $\min ), 95^{\circ} \mathrm{C}(10 \mathrm{~min})$, followed by 40 cycles of $95^{\circ} \mathrm{C}(15$ s) and $60^{\circ} \mathrm{C}(1 \mathrm{~min})$.

\section{Western blotting analysis}

Whole-cell, cytoplasmic, and nuclear extracts from HT29 and Colo320DM cells were prepared using a Nuclear Extract kit (Active Motif, Tokyo, Japan) according to the manufacturer's instructions. The protein concentration was determined using a Pierce BCA Protein Assay Kit (Thermo Scientific, Waltham, MA). Wholecell lysates containing $5 \mu \mathrm{g}$ of protein from HT29 cells and $12.5 \mu \mathrm{g}$ of protein from Colo320DM cells were loaded in each lane, run on a NuPAGE 4\%-12\% Bis-Tris gel (Invitrogen, Carlsbad, CA), and transferred onto PVDF iBlot Gel Transfer Stacks (Invitrogen). After blotting, membranes were blocked in Tris-buffered saline containing $0.05 \%$ Tween-20 (TBS-T) and $1 \%$ non-fat dried milk for $1 \mathrm{~h}$. After blocking, membranes were probed overnight at $4{ }^{\circ} \mathrm{C}$ with a rat monoclonal antibody against Keap1 (dilution 1:5,000; clone\#144), a rabbit polyclonal antibody against Nrf2 (1:200; Santa Cruz, \#sc-722), a mouse monoclonal antibody against NQO-1 (1:1,000; Santa Cruz Biotechnology, \#sc-32793), and a mouse monoclonal antibody against AKR1C1 (1:1,000; ATGen,\#ATGA0201). Membranes were washed four times (10 min per wash) with antibody dilution buffer and then incubated with goat anti-rabbit IgG (1:2,000; Santa Cruz Biotechnology) for $1 \mathrm{~h}$ at room temperature. A rabbit monoclonal antibody against $\beta$-actin $(1: 2,000$; Cell Signaling Technologies, Danvers, MA) and a mouse monoclonal Antibody against histone H1 (1:500; Santa Cruz Biotechnology, \#sc-8030) were used as controls. After extensive washing $(4 \times 10 \mathrm{~min}$ with TBS-T), antibody detection was performed with SuperSignal West Pico Chemiluminescent Substrate Kits (Pierce, Rockford, IL).

\section{Statistical analysis}

Data are presented as the means \pm standard deviation. Student's $t$ test was used to assess the significance of three independent experiments. In all analyses, $P<0.05$ was taken to indicate statistical significance.

\section{Results}

\section{Genetic alteration of KEAP1 in CRC cell lines}

As KEAP1 gene mutations have been reported in other types of human cancer, we sequenced all protein-coding exons in $10 \mathrm{CRC}$ cell lines. We detected a C-to- $\mathrm{T}$ transition (G157G) in exon 2 of LoVo cells, a C-to-G transition (L470L) in exon 4 of LoVo, DLD-1, TT1TKB, HCT15, and CW-2 cells, and a C-to-T transition (Y537Y) in exon 5 of CW-2 cells. All mutations were single-nucleotide polymorphisms and had been reported previously. No missense or nonsense mutations were observed.

\section{Analysis of the methylation status of the KEAP1 promoter} region in $10 \mathrm{CRC}$ cell lines

The KEAP1 promoter region was hypermethylated in lung cancer cell lines and lung cancer tissues, as reported previously by Wang et al. [20]. They reported that the P1 region, including 12 CpGs (-291 to -89), was heavily hypermethylated in the $\mathrm{CpG}$ islands around the transcriptional initiation site of KEAP1. Therefore, we investigated the methylation status of the $\mathrm{P} 1$ region in KEAP1 using MSP and BSP primers designed as shown in Figure 1. MSP analysis indicated that the P1 region was hypermethylated in HT29, WiDr, LoVo, DLD-1, SW480, TT1TKB, HCT15, and CW-2 cells, but not in SW837 or Colo320DM (Figure 2A). Furthermore, we determined the methylation status of each of the 12 $\mathrm{CpG}$ dinucleotide sites in the $\mathrm{P} 1$ region by $\mathrm{BSP}$. As shown in Figure 2B, most of CpG sites were methylated in HT29, WiDr, LoVo, DLD-1, SW480, TT1TKB, HCT15, and CW-2, but not in SW837 or Colo320DM. Representative results of methylation analysis of CpG islands in the promoter region of the KEAP1 gene are shown in Figure 2B. All cytosines in the P1 region were converted to thymidine in Colo320DM cells, although in HT29 cells the 5'-methylcytosines of CpG sites remained as cytosines. In contrast, both cytosines and thymidines in the $5^{\prime}$-methylcytosines of CpG sites were observed in HCT15 cells. Aberrant hypermethylation in the KEAP1 promoter region was frequently observed in human CRC cell lines.

\section{Association between KEAP1 methylation and KEAP1 mRNA expression}

To examine the effects of KEAP1 methylation on its mRNA expression level, we performed real-time RTPCR of KEAP1 mRNA as shown in Figure 3A. Cell lines with methylated KEAP1 (HT29, WiDr, LoVo, DLD-1, SW480, TT1TKB, HCT15, and CW-2) exhibited lower levels of KEAP1 mRNA expression compared with the unmethylated cell lines SW837 and Colo320DM. 
To determine whether expression of KEAP1 mRNA is epigenetically downregulated, expression of KEAP1 mRNA was measured after treatment with the demethylating agent 5 -Aza-dC at $10 \mu \mathrm{M}$ for 4 days and/or the reversible histone deacetylase inhibitor TSA at $1 \mu \mathrm{M}$ for $24 \mathrm{~h}$ in HT29 cells. The expression of KEAP1 mRNA was markedly increased after 5-Aza-dC and TSA treatment in the methylated cell line HT29, but no changes were observed in KEAP1 mRNA expression level in the unmethylated cell line Colo320DM (Figure 3B). MSP analysis showed that methylation of the keap1 promoter in HT29 cells was reversed after 5-Aza-dC and TSA treatment (Figure 3B). These observations suggest that epigenetic alterations regulate Keap1 expression in CRC cell lines.

\section{Protein levels of Keap1 and Nrf2}

To further examine whether Keap1 protein levels are different between methylated and unmethylated cells, we performed Western blotting analysis. The Keap1 protein level was reduced in HT29 cells, compared with that in Colo320DM cells, as shown in Figure 3C (left). Keap1 protein expression in the methylated cell line HT29 was reversed by treatment with 5-Aza-dC and TSA, but was unchanged in the unmethylated cell line Colo320DM (Figure 4C), mirroring similar changes in
KEAP1 mRNA expression. In addition, Nrf2 protein clearly accumulated in the nuclear fraction of HT29 cells, as compared to its level in Colo320DM, whereas Nrf2 protein levels in cytoplasmic fractions were equivalent in these two cell lines (Figure 3C, right). Nrf2 protein accumulation in HT29 cells was reduced by demethylation (Figure 4C).

\section{NQO1 and AKR1C1 mRNA and protein levels}

We measured NQO1 and AKR1C1 mRNA levels at different time points after treatment with $\mathrm{t}-\mathrm{BHQ}$, an activator of the Nrf2-ARE pathway, at a concentration of100 $\mu \mathrm{M}$. NQO1 and AKR1C1 expression levels were higher in the methylated cell line HT29 than in the unmethylated cell line Colo320DM without stimulation (Figures $4 \mathrm{~A}$ and $4 \mathrm{~B}$ left). Furthermore, $\mathrm{t}-\mathrm{BHQ}$ treatment significantly increased NQO-1 and AKR1C1 mRNA levels in HT29 cells. AKR1C1 mRNA was below the limit of detection both at baseline and after stimulation in Colo320DM cells. The expression of NQO1 and AKR1C1 mRNA in the methylated cell line HT29 was reversed after treatment with 5-Aza-dC and TSA (Figures $4 \mathrm{~A}$ and $4 \mathrm{~B}$ (right)). NQO-1 and AKR1C1 proteins were overexpressed in methylated HT 29 cells, but their levels were reduced after demethylation (Figure 4C). 
A

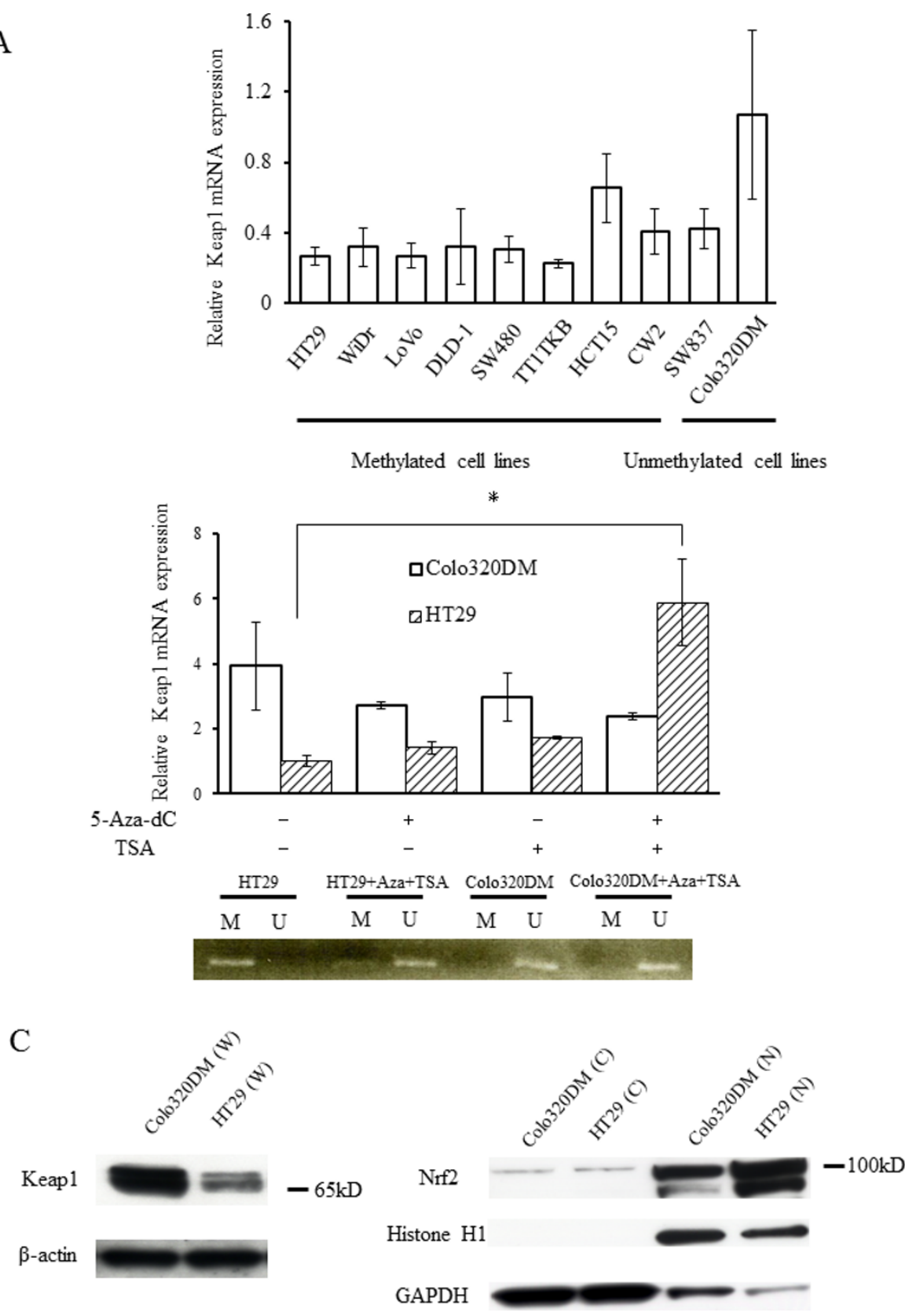

B

Figure 3 KEAP1 and Nrf2 expression. (A) KEAP1 mRNA expression in 10 CRC cell lines was evaluated by real-time PCR. The expression level in Colo320DM cells was arbitrarily designated as 1. Columns, mean $(n=3)$; bars, standard deviation (SD). (B, upper) KEAP1 mRNA levels in HT29 cells (methylated) and Colo320DM cells (unmethylated) were analyzed by real-time PCR after treatment with 5-Aza-dC, TSA, and 5-Aza-dC + TSA. The expression level in HT29 cells was arbitrarily designated as 1. Columns, mean $(n=3)$; bars, SD. ${ }^{*} P<0.05$. (B, lower) MSP analysis of in HT29 cells and Colo320DM treated with 5-Aza-dC + TSA. M, methylation-specific primer; U, non-methylation-specific primer. (C) Western blot analysis of Keap1 and Nrf2 in methylated and unmethylated colon cancer cells. Whole-cell extracts (W), cytosolic extracts (C), and nuclear extracts (N) were prepared from Colo320DM and HT29 cells. Extracts were stained with antibody to Keap1 or Nrf2A. $\beta$-Actin, histone H1, and GAPDH antibodies were used as loading controls for whole-cell, cytosolic, and nuclear fractions, respectively.

\section{Detection of KEAP1 methylation using MSP in surgical} samples and association between methylation status and clinicopathological features in CRC

The methylation status of each sample was confirmed by MSP and BSP. Representative MSP products for
KEAP1 in tumor tissues and normal tissues are shown in Figure 5A. Representative results of MSP sequence analysis of tumor tissues are presented in Figure 5B. Aberrant promoter methylation of KEAP1 was detected in $21 / 40$ (53\%) tumor tissues and 10/40 (25\%) normal 


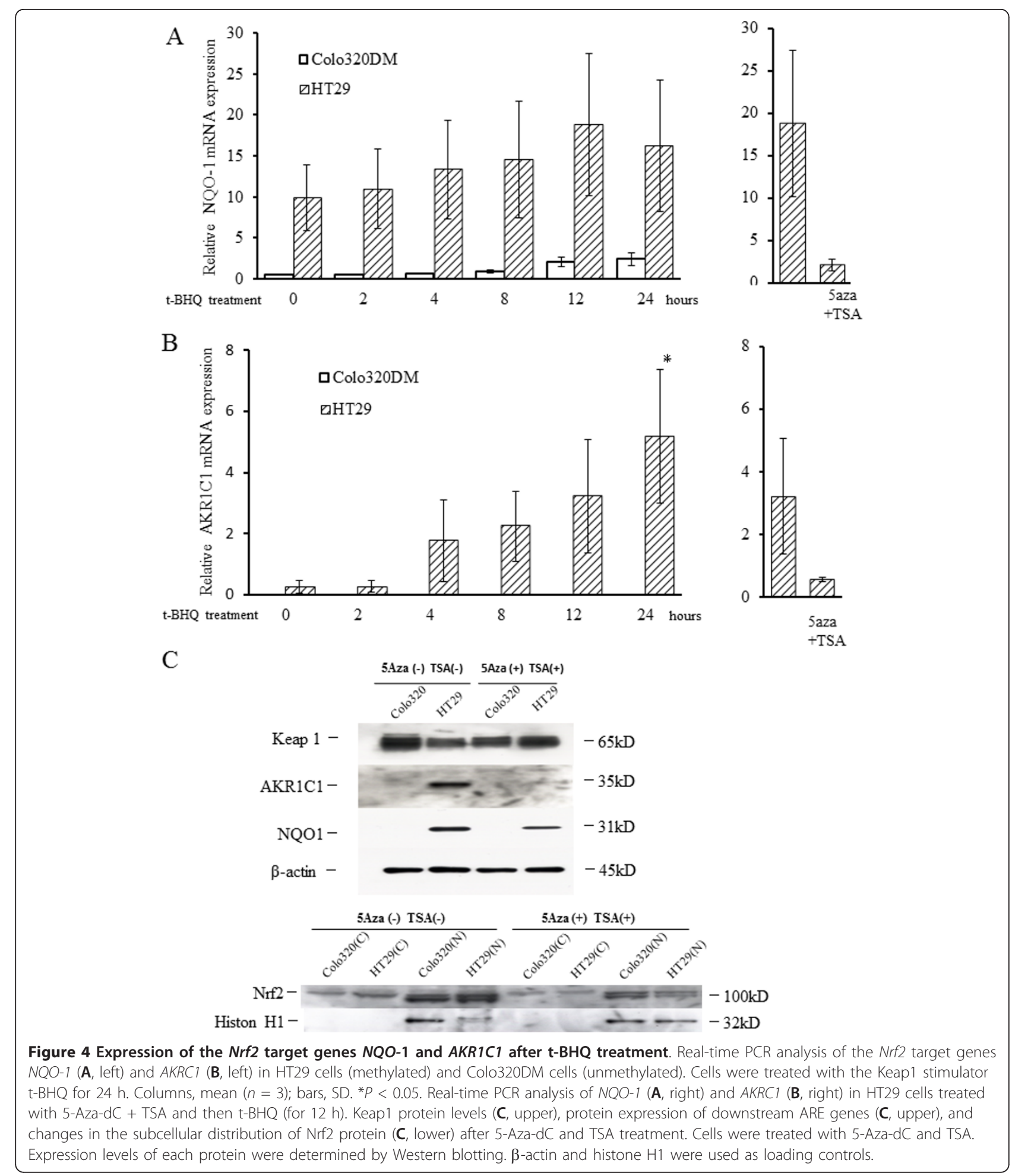

mucosal specimens (Table 2). Compared with normal mucosa, the methylation of KEAP1 was more prominent in tumor tissues $(P=0.001)$. We performed statistical analyses to determine whether the KEAP1 methylation status of colorectal tumor samples is associated with the clinicopathological features of CRC patients. In the tumor tissues, methylation of KEAP1 was not associated with any clinicopathological features, such as primary site location, differentiation, gender, Duke's stage, clinical stage, age, lymph node 
A

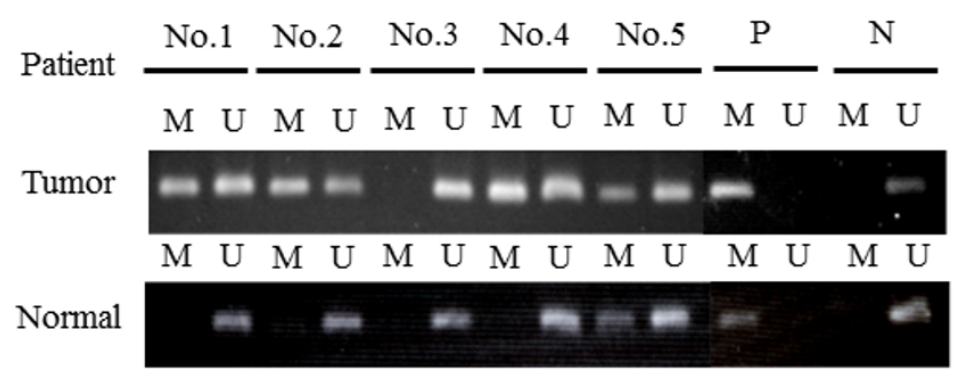

$\mathrm{B}$

TC GG ATTA TGAGGT CGGCGTTGTGCGTT GTTAAAAGG AGAATAGTAG ATGGT GGC G CG TA G TTT CGCG AGG

No.1

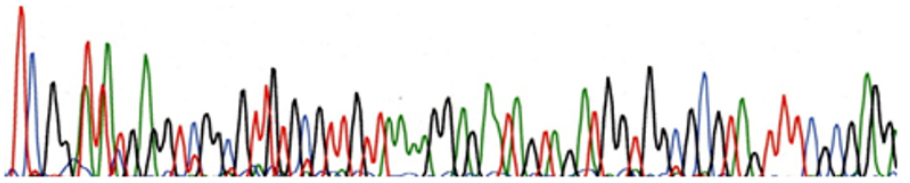

TGGATTATGAGGTTGG TGTT GT GTGT TG TTAAAAGGAGAA TAGTAGAT GGT GGT GT G TA G T TT TG T G AGG

No.3

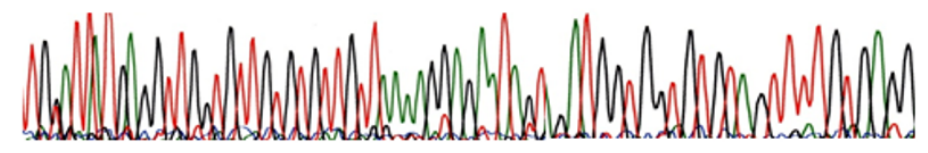

HT29

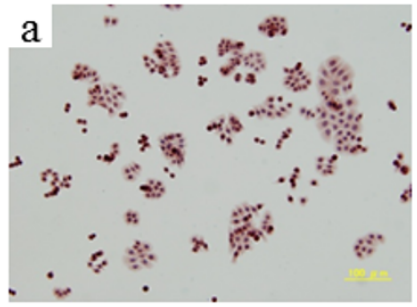

Tissue sample

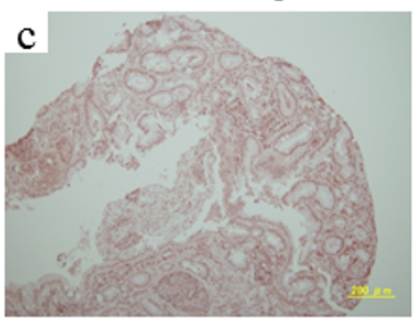

Colo320

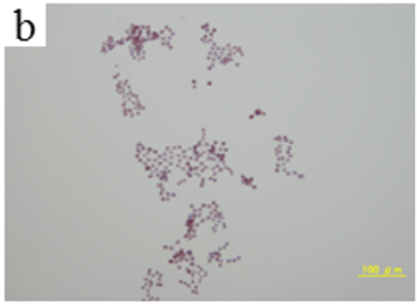

Tissue sample

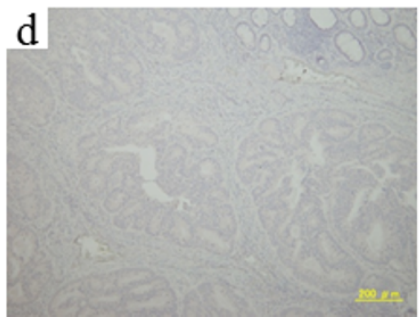

Figure 5 Methylation of the KEAP1 promoter in CRC tissue samples. (A) MSP for the KEAP1 promoter was performed using bisulfitemodified DNA from 40 CRC tissues and adjacent normal colorectal tissues. MSP results from 10 patients are shown. M: MSP of methylationspecific primers; U: MSP of non-methylation-specific primers; P: positive methylated DNA control; N: negative unmethylated DNA control. (B) Representative results of MSP sequence analysis of tumor tissues. The methylation status of the KEAP1 promoter region in patient tumor tissues was determined using methylation-specific primers (upper), and non-methylation-specific primers (lower). Methylation is indicated in red. (C) Expression and subcellular localization of Nrf2. Nrf2 was more highly expressed in HT29 cells (a) and a methylated tissue sample (c) compared with Colo320 cells (b) and a non-methylated tissue sample (d). Expression and localization of Nrf2 was studied using an anti-human Nrf2 antibody.

metastasis, and serum concentration of carcinoembryonic antigen (CEA) (data not shown). Additionally, we analyzed methylated HT29 cells and tumor samples by immunohistochemistry using an anti-human Nrf2 antibody. As shown in Figure 5C, strong expression of Nrf2 protein was detected in the nuclei of HT29 cells and in a methylated tissue sample. This observation indicates that promoter methylation of the KEAP1 
Table 2 KEAP1 promoter methylation frequency in colorectal cancer and adjacent normal mucosa

\begin{tabular}{llll}
\hline & & \multicolumn{2}{l}{ Methylation status } \\
\cline { 3 - 4 } Variable & N & Present & Absent \\
\hline Tumor tissue & 40 & $21(53 \%)$ & $19(47 \%)$ \\
Adjacent normal mucosa & 40 & $10(25 \%)$ & $30(75 \%)$ \\
\hline & & & $P<0.05$ \\
\hline
\end{tabular}

gene enables Nrf2 to translocate from the cytoplasm to the nucleus.

\section{Discussion and conclusions}

We found frequent hypermethylation of the KEAP1 promoter region in human CRC cell lines. This hypermethylation of KEAP1 resulted in reductions in KEAP1 mRNA and protein expression, upregulation of Nrf2 activity, and thus overexpression of downstream genes, such as NQO-1 and $A K R 1 C 1$. We also observed aberrant methylation of KEAP1 in human CRC tissues. This is the first report discussing activation of Keap1/Nrf2 signaling by KEAP1 hypermethylation in CRC.

Loss of Keap1 function has been reported associated with KEAP1 gene mutations in tumor tissue samples from lung, gall bladder, breast, and prostate cancer $[15,18,19,21]$. We found only synonymous mutations consisting of a C-to-T transition with G157G in exon 2, a T-to-C transition of L471L in the DGR4 domain, and a C-to- $\mathrm{T}$ transition with Y537Y in the DGR5 domain in CRC cell lines. However, these mutations were singlenucleotide polymorphisms. Frequent KEAP1 gene mutations were reported in human non-small cell lung cancer (NSCLC) [15]. All mutations were within highly conserved amino acid residues located in the Kelch or intervening region domain of the Keap1 protein, suggesting that these mutations were likely to abolish Keap1 repressor activity against Nrf2. In addition, C23Y mutation in the $\mathrm{N}$-terminal domain of Keap1 has been reported to have impaired ability to repress Nrf2 activity due to its inability to stimulate the ubiquitylation and degradation of Nrf2 in breast cancer [19]. A C-to-T transition with $\mathrm{T} 314 \mathrm{M}$ and a $\mathrm{T}$-to- $\mathrm{C}$ transition with $\mathrm{Y} 255 \mathrm{H}$ were detected in six prostatic cancer cell lines [21]. Shibata et al. also reported mutations of KEAP1 in biliary tract cancer tissue [18]. These changes are in the central intervening region of Keap 1 and alter highly conserved amino acids.

Another mechanism of impaired Keap1 activity is hypermethylation of KEAP1. We found that 8 of 10 CRC cell lines had methylated CpG islands in the promoter region of the KEAP1 gene where methylation was found in other types of cancer [20,22,23]. Hypermethylation of KEAP1 resulted in decreased mRNA expression, which was confirmed by the increase in KEAP1 mRNA expression by combined treatment with the DNA methyltransferase inhibitor 5-Aza-dC and the histone deacetylase inhibitor TSA (Figure 4C). Hypermethylation of KEAP1 caused final stimulation of Nrf2 target genes. However, the reason for the expression of KEAP1 mRNA being lower in unmethylated SW837 cells than in methylated HCT15 cells is unknown. Wang et al. investigated three lung cancer cell lines and five tumor samples, and found frequent hypermethylation of the CpG islands in the promoter region of KEAP 1 and reduced levels of KEAP1 mRNA expression. In contrast, a normal bronchial cell line had clearly less methylation of the KEAP1 promoter region and elevated mRNA expression [20]. Hypermethylation of KEAP1 found in prostate cancer also stimulated the Nrf2 signal [21].

Biological effects of constitutive Nrf2 activation by Keap1 dysfunction due to mutations or low-level expression by hypermethylation have been reported previously $[18,23,24]$. Constitutive expression of the cytoprotective gene by Nrf2 activation in lung cancer cells led to chemotherapy resistance [23]. Nrf2 activation also stimulated growth of lung cancer cells. Nrf2 activation by KEAP1 mutation or hypermethylation of promoter CpG islands causes radioresistance and promotes tumor growth in prostatic cancer [21]. In the present study, we observed accumulation of Nrf2 protein in the nuclei in methylated HT29 cells, and overexpression of phase II detoxifying enzymes NQO-1 and AKR1C1 both at baseline and after $\mathrm{t}-\mathrm{BHQ}$ stimulation. These reports indicate that KEAP1 functions as a tumor suppressor gene in human tumors. Although we did not evaluate the biological effects of activated Nrf2, we assume that CRC cells with KEAP1 gene hypermethylation may be resistant to chemotherapeutic agents and show upregulated cell growth, as reported in other types of cancer.

There have been only two previous reports regarding Keap1/Nrf2 1 in CRC cells [24,25]. Activation of the Keap1/Nrf2 signaling pathway mediates protective responses to mitigate nitric oxide (NO)-induced damage and may contribute to the resistance of CRC cells to NO-induced cytotoxicity [24]. Arlt et al. reported that Nrf2 activity is elevated in colon cancer, accounting for overexpression of the proteasome subunit proteins and thus for increased proteasome activity [25]. Conversely, small interfering RNA-mediated Nrf2 knockdown decreased their expression and reduced proteasome activity, thus indicating that Nrf2 is related to colorectal carcinogenesis. This Nrf2 activation may be due to the low level of Keap1 expression due to hypermethylation, as found in the present study.

Biological effects that activate Nrf2 signaling prompted us to study the relationship between the status of Keap1/Nrf2 signaling and clinicopathological features of the tumors. Type II endometrial cancer, which is mostly 
malignant and is associated with a poor prognosis among gynecological malignancies, shows elevated Nrf2 protein expression, whereas benign tumors and type I endometrial cancer do not [26]. On immunohistochemical analysis of human NSCLC, increased Nrf2 expression and low or absent Keap1 expression were associated with worse survival [27]. In contrast, the prognosis of malignant glioma was better among patients with than among those without a methylated KEAP1 promoter region [22]. Although we did not investigate the prognosis of patients with $\mathrm{CRC}$, further studies are needed to understand the role of Keap1/Nrf2 signaling in human CRC.

In conclusion, the results of the present study revealed hypermethylation of the KEAP1 promoter region in human CRC, leading to downregulation of KEAP1 mRNA expression, thus activating Nrf2 and expression of its downstream target genes. Cancerous tissues exhibited more frequent methylation of KEAP1 than normal tissue in surgically resected CRC specimens.

\section{Acknowledgements}

This work was supported in part by Grants-in-Aid for Scientific Research from the Ministry of Education, Science, Sports, Culture and Technology, Japan (Nos. 16022206 and 20390229).

\section{Author details}

'Department of Medical Oncology, Hirosaki University Graduate School of Medicine, 5 Zaifu-cho, Hirosaki 036-8562, Japan. ${ }^{2}$ Department of Gastroenterology and Hematology, Hirosaki University Graduate School of Medicine, 5 Zaifu-cho, Hirosaki 036-8562, Japan. ${ }^{3}$ Department of Pathology and Bioscience, Hirosaki University Graduate School of Medicine, 5 Zaifu-cho, Hirosaki 036-8562, Japan. ${ }^{4}$ Department of Stress Response Science, Hirosaki University Graduate School of Medicine, 5 Zaifu-cho, Hirosaki 036-8562, Japan. ${ }^{5}$ Department of Rheumatology and Immunology, Shengjing Hospital of China Medical University, Shenyang, China.

\section{Authors' contributions}

$H N, T T, Z Q, Y X, S R$, and $M J$ performed experiments and summarized the data. IJ, IA, IK, FS, and SY designed the experiments. HN, TT, and SY wrote the paper; all authors have read and approved the final manuscript.

\section{Competing interests}

The authors declare that they have no competing interests.

Received: 10 August 2011 Accepted: 13 February 2012 Published: 13 February 2012

\section{References}

1. Jemal A, Siegel R, Ward E, Hao Y, Xu J, Thun MJ: Cancer statistics. CA Cancer J Clin 2009, 59:225-249.

2. Zhang DD: Mechanistic studies of the Nrf2-Keap1 signaling pathway. Drug Metab Rev 2006, 38:769-789.

3. Cho HY, Reddy SP, Debiase A, Yamamoto M, Kleeberger SR: Gene expression profiling of NRF2-mediated protection against oxidative injury. Free Radic Biol Med 2005, 38:325-343.

4. Kobayashi A, Kang MI, Watai Y, Tong Kl, Shibata T, Uchida K, Yamamoto M: Oxidative and electrophilic stresses activate Nrf2 through inhibition of ubiquitination activity of Keap1. Mol Cell Biol 2006, 26:221-229.

5. Moi P, Chan K, Asunis I, Cao A, Kan YW: Isolation of NF-E2-related factor 2 (Nrf2), a NF-E2-like basic leucine zipper transcriptional activator that binds to the tandem NF-E2/AP1 repeat of the beta-globin locus control region. Proc Natl Acad Sci USA 1994, 91:9926-9930.
6. Itoh K, Igarashi K, Hayashi N, Nishizawa M, Yamamoto M: Cloning and characterization of a novel erythroid cell-derived CNC family transcription factor heterodimerizing with the small Maf family proteins. Mol Cell Biol 1995, 15:4184-4193.

7. Itoh K, Chiba T, Takahashi S, Ishii T, Igarashi K, Katoh Y, Oyake T, Hayashi N, Satoh K, Hatayama I, Yamamoto M, Nabeshima Y: An Nrf2/small Maf heterodimer mediates the induction of phase II detoxifying enzyme genes through antioxidant response elements. Biochem Biophys Res Commun 1997, 236:313-322.

8. Itoh K, Wakabayashi N, Katoh Y, Ishii T, Igarashi K, Engel JD, Yamamoto M: Keap1 represses nuclear activation of antioxidant responsive elements by Nrf2 through binding to the amino-terminal Neh2 domain. Genes Dev 1999, 13:76-86.

9. Zhang DD, Lo SC, Cross JV, Templeton DJ, Hannink M: Keap1 is a redoxregulated substrate adaptor protein for a Cul3-dependent ubiquitin ligase complex. Mol Cell Biol 2004, 24:10941-10953.

10. Itoh S, Taketomi A, Harimoto N, Tsujita E, Rikimaru T, Shirabe K, Shimada M, Maehara $Y$ : Antineoplastic effects of gamma linoleic acid on hepatocellular carcinoma cell lines. J Clin Biochem Nutr 2010, 47:81-90.

11. Cullinan SB, Gordan JD, Jin J, Harper JW, Diehl JA: The Keap1-BTB protein is an adaptor that bridges Nrf2 to a Cul3-based E3 ligase: oxidative stress sensing by a Cul3-Keap1 ligase. Mol Cell Biol 2004, 24:8477-8486.

12. Furukawa $M$, Xiong Y: BTB protein Keap1 targets antioxidant transcription factor Nrf2 for ubiquitination by the cullin 3-Roc1 ligase. Mol Cell Biol 2005, 25:162-171.

13. Dinkova-Kostova AT, Holtzclaw WD, Cole RN, Itoh K, Wakabayashi N, Katoh Y, Yamamoto M, Talalay P: Direct evidence that sulfhydryl groups of Keap1 are the sensors regulating induction of phase 2 enzymes that protect against carcinogens and oxidants. Proc Natl Acad Sci USA 2002, 99:11908-11913.

14. Wakabayashi N, Dinkova-Kostova AT, Holtzclaw WD, Kang MI, Kobayashi A, Yamamoto M, Kensler TW, Talalay P: Protection against electrophile and oxidant stress by induction of the phase 2 response: fate of cysteines of the Keap1 sensor modified by inducers. Proc Natl Acad Sci USA 2004, 101:2040-2045

15. Singh A, Misra V, Thimmulappa RK, Lee H, Ames S, Hoque MO, Herman JG, Baylin SB, Sidransky D, Gabrielson E, Brock MV, Biswal S: Dysfunctional KEAP1-NRF2 interaction in non-small-cell lung cancer. PLoS Med 2006, 3:1865-1875.

16. Padmanabhan B, Tong Kl, Ohta T, Nakamura Y, Scharlock M, Ohtsuji M, Kang MI, Kobayashi A, Yokoyama S, Yamamoto M: Structural basis for defects of Keap1 activity provoked by its point mutations in lung cancer. Mol Cell 2006, 21:689-700.

17. Shibata T, Ohta T, Tong Kl, Kokubu A, Odogawa R, Tsuta K, Asamura H, Yamamoto M, Hirohashi S: Cancer related mutations in NRF2 impair its recognition by Keap1-Cul3 E3 ligase and promote malignancy. Proc Natl Acad Sci USA 2008, 105:13568-13573.

18. Shibata T, Kokubu A, Gotoh M, Ojima H, Ohta T, Yamamoto M, Hirohashi S: Genetic alteration of Keap1 confers constitutive Nrf2 activation and resistance to chemotherapy in gallbladder cancer. Gastroenterology 2008, 135:1358-1368.

19. Nioi P, Nguyen T: A mutation of Keap1 found in breast cancer impairs its ability to repress Nrf2 activity. Biochem Biophys Res Commun 2007, 362:816-821.

20. Wang R, An J, Ji F, Jiao H, Sun H, Zhou D: Hypermethylation of the Keap1 gene in human lung cancer cell lines and lung cancer tissues. Biochem Biophys Res Commun 2008, 373:151-154.

21. Zhang P, Singh A, Yegnasubramanian S, Esopi D, Kombairaju P, Bodas M, Wu H, Bova SG, Biswal S: Loss of Kelch-like ECH-associated protein 1 function in prostate cancer cells causes chemoresistance and radioresistance and promotes tumor growth. Mol Cancer Ther 2010, 9:336-346.

22. Muscarella LA, Barbano R, D'Angelo V, Copetti M, Coco M, Balsamo T, la Torre A, Notarangelo A, Troiano M, Parisi S, Icolaro N, Catapano D, Valori VM, Pellegrini F, Merla G, Carella M, Fazio VM, Parrella P: Regulation of KEAP1 expression by promoter methylation in malignant gliomas and association with patient's outcome. Epigenetics 2011, 6:317-325.

23. Ohta T, lijima K, Miyamoto M, Nakahara I, Tanaka H, Ohtsuji M, Suzuki T, Kobayashi A, Yokota J, Sakiyama T, Shibata T, Yamamoto M, Hirohashi S: Loss of Keap1 function activates Nrf2 and provides advantages for lung cancer cell growth. Cancer Res 2008, 68:1303-1309. 
24. Li CQ, Kim MY, Godoy LC, Thiantanawat A, Trudel LJ, Wogan GN: Nitric oxide activation of Keap1/Nrf2 signaling in human colon carcinoma cells. Proc Natl Acad Sci USA 2009, 106:14547-14551.

25. Arlt A, Bauer I, Schafmayer C, Tepel J, Müerköster SS, Brosch M, Röder C, Kalthoff H, Hampe J, Moyer MP, Fölsch UR, Schäfer H: Increased proteasome subunit protein expression and proteasome activity in colon cancer relate to an enhanced activation of nuclear factor E2related factor 2 (Nrf2). Oncogene 2009, 28:3983-3996.

26. Jiang $T$, Chen N, Zhao F, Wang XJ, Kong B, Zheng W, Zhang DD: High levels of Nrf2 determine chemoresistance in type II endometrial cancer. Cancer Res 2010, 70:5486-5496.

27. Solis LM, Behrens C, Dong W, Suraokar M, Ozburn NC, Moran CA, Corvalan AH, Biswal S, Swisher SG, Bekele BN, Minna JD, Stewart DJ, Wistuba II: Nrf2 and Keap1 abnormalities in non-small cell lung carcinoma and association with clinicopathologic features. Clin Cancer Res 2010, 16:3743-3753.

Pre-publication history

The pre-publication history for this paper can be accessed here: http://www.biomedcentral.com/1471-2407/12/66/prepub

doi:10.1186/1471-2407-12-66

Cite this article as: Hanada et al.: Methylation of the KEAP1 gene promoter region in human colorectal cancer. BMC Cancer 2012 12:66.

\section{Submit your next manuscript to BioMed Central} and take full advantage of:

- Convenient online submission

- Thorough peer review

- No space constraints or color figure charges

- Immediate publication on acceptance

- Inclusion in PubMed, CAS, Scopus and Google Scholar

- Research which is freely available for redistribution

Submit your manuscript at www.biomedcentral.com/submit 